\title{
Density Functional Theory Study of the $\beta$-Carotene Radical Cation and Deprotonated Radicals
}

Yunlong Gao, ${ }^{\mathrm{a}}$ A. Ligia Focsan, ${ }^{\mathrm{b}}$ Lowell D. Kispert, ${ }^{\mathrm{b}}{ }^{\mathrm{b}}$ and David Dixon* ${ }^{\mathrm{b}}$

${ }^{a}$ Key Lab of Analytical Chemistry for Life Science, Department of Chemistry, Nanjing University, Nanjing, 210093, P.R. China

b Department of Chemistry, Shelby Hall, Box 870336, The University of Alabama, Tuscaloosa, AL 35487-0336

Supporting Information: Calculated hyperfine coupling constants ( $\mathrm{MHz}$ ) of protons, calculated spin density distribution, total electronic energy, and optimized geometry parameters for $\mathrm{Car}^{+}{ }^{+}$Car $^{+}{ }^{+}$in water, \#Car·(5), \#Car·(9) and \#Car· (13) with different DFT methods. Hyperfine coupling constants used in the simulations calculated at the B3LYP/TZP(Ahlrichs)// B3LYP/6-31G** level. 
TABLE S1: Calculated Hyperfine Coupling Tensors (MHz) of Methyl Protons and $\mathrm{CH}_{2}$ Protons (from loss of the proton from $\mathrm{Car}^{+}$) in $\mathrm{Car}+{ }^{+}, \mathrm{Car}+$ in water, \#Car·(5), \#Car.(9) and \#Car· (13) using B3PW91/TZP(Ahlrichs)//B3LYP/6-31G**.

\begin{tabular}{|c|c|c|c|c|c|c|}
\hline Position & Parameter & $\mathrm{Car}^{+}$ & $\begin{array}{c}\mathrm{Car}^{+} \text {in } \\
\text { water }\end{array}$ & $\# \operatorname{Car} \cdot(5)$ & $\# \operatorname{Car} \cdot(9)$ & \#Car.(13) \\
\hline \multirow[t]{4}{*}{$5^{\prime}$} & $\mathrm{A}_{11}$ & 5.25 & 4.65 & 1.74 & 2.04 & 2.64 \\
\hline & $\mathrm{A}_{22}$ & 5.70 & 5.09 & 2.15 & 2.45 & 3.14 \\
\hline & $\mathrm{A}_{33}$ & 6.87 & 6.18 & 2.86 & 3.25 & 4.12 \\
\hline & $\mathbf{A}_{\text {iso }}$ & 5.94 & 5.31 & 2.25 & 2.58 & 3.30 \\
\hline \multirow[t]{4}{*}{$9^{\prime}$} & $\mathrm{A}_{11}$ & 6.12 & 6.78 & 7.75 & 8.34 & 10.34 \\
\hline & $\mathrm{A}_{22}$ & 6.94 & 7.64 & 8.74 & 9.42 & 11.59 \\
\hline & $\mathrm{A}_{33}$ & 8.15 & 8.89 & 10.06 & 10.83 & 13.26 \\
\hline & $\mathbf{A}_{\text {iso }}$ & 7.07 & 7.77 & 8.85 & 9.53 & 11.73 \\
\hline \multirow[t]{4}{*}{ 13' } & $\mathrm{A}_{11}$ & 3.72 & 4.40 & 11.40 & 12.00 & 13.84 \\
\hline & $\mathrm{A}_{22}$ & 4.50 & 5.21 & 12.75 & 13.43 & 15.41 \\
\hline & $\mathrm{A}_{33}$ & 5.34 & 6.10 & 14.37 & 15.13 & 17.34 \\
\hline & $\mathbf{A}_{\text {iso }}$ & 4.52 & 5.24 & 12.84 & 13.52 & 15.53 \\
\hline \multirow[t]{4}{*}{5} & $\mathrm{~A}_{11}$ & 5.25 & 4.65 & -2.38 & -0.41 & -0.29 \\
\hline & $\mathrm{A}_{22}$ & 5.70 & 5.09 & -1.44 & -0.32 & -0.19 \\
\hline & $A_{33}$ & 6.87 & 6.18 & -0.39 & 0.08 & -0.06 \\
\hline & $\mathbf{A}_{\text {iso }}$ & 5.94 & 5.31 & -1.40 & -0.22 & -0.18 \\
\hline \multirow[t]{4}{*}{9} & $\mathrm{~A}_{11}$ & 6.12 & 6.78 & -3.93 & -11.45 & -1.25 \\
\hline & $\mathrm{A}_{22}$ & 6.94 & 7.64 & -3.74 & -7.18 & -1.01 \\
\hline & $\mathrm{A}_{33}$ & 8.15 & 8.89 & -2.98 & -2.38 & -0.71 \\
\hline & $\mathbf{A}_{\text {iso }}$ & 7.07 & 7.77 & -3.55 & -7.00 & -0.99 \\
\hline \multirow[t]{4}{*}{13} & $\mathrm{~A}_{11}$ & 3.72 & 4.40 & -6.51 & -6.35 & -22.35 \\
\hline & $\mathrm{A}_{22}$ & 4.50 & 5.21 & -6.30 & -6.03 & -14.14 \\
\hline & $\mathrm{A}_{33}$ & 5.34 & 6.10 & -5.40 & -5.17 & -5.27 \\
\hline & $\mathbf{A}_{\text {iso }}$ & 4.52 & 5.24 & -6.07 & -5.85 & -13.92 \\
\hline
\end{tabular}


TABLE S2: Calculated Hyperfine Coupling Constants $(\mathrm{MHz})$ of $\alpha$-protons in $\mathrm{Car}^{+}$, $\mathrm{Car}^{+}{ }^{+}$in water, \#Car·(5), \#Car·(9) and \#Car· (13) using B3LYP/TZP(Ahlrichs)//B3LYP/6$31 \mathrm{G}^{* *}$.

\begin{tabular}{|c|c|c|c|c|c|}
\hline Position & Car $^{+}$ & $\begin{array}{l}\mathrm{Car}^{+} \text {in } \\
\text { water }\end{array}$ & \#Car·(5) & \#Car·(9) & \#Car·(13) \\
\hline \multirow[t]{2}{*}{4} & 9.19 & 8.33 & -0.99 & -0.32 & -0.42 \\
\hline & 12.06 & 10.70 & -0.01 & -0.25 & -0.41 \\
\hline \multirow[t]{2}{*}{$4^{\prime}$} & 12.06 & 10.71 & 4.06 & 4.58 & 6.18 \\
\hline & 9.19 & 8.33 & 4.14 & 4.84 & 6.64 \\
\hline 7 & -7.66 & -7.80 & 2.26 & 0.72 & 0.81 \\
\hline $7^{\prime}$ & -7.66 & -7.79 & -7.26 & -8.13 & -11.94 \\
\hline 8 & 1.45 & 1.71 & -6.55 & -1.29 & -0.27 \\
\hline $\mathbf{8}^{\prime}$ & 1.45 & 1.70 & 2.09 & 2.41 & 4.63 \\
\hline 10 & 0.34 & 0.81 & -8.78 & -10.08 & -0.53 \\
\hline $10^{\prime}$ & 0.34 & 0.81 & 3.40 & 3.84 & 7.05 \\
\hline 11 & -6.43 & -6.99 & 3.67 & 3.44 & 2.03 \\
\hline 11' & -6.43 & -6.98 & -11.45 & -12.54 & -16.88 \\
\hline 12 & 0.44 & 0.80 & -10.5 & -10.43 & -2.97 \\
\hline $12^{\prime}$ & 0.44 & 0.80 & 4.58 & 5.14 & 8.88 \\
\hline 14 & -1.81 & -1.65 & -12.59 & -13.11 & -18.20 \\
\hline $14^{\prime}$ & -1.82 & -1.66 & 5.19 & 5.60 & 8.94 \\
\hline 15 & -3.10 & -3.28 & 5.21 & 5.48 & 7.93 \\
\hline $15^{\prime}$ & -3.10 & -3.27 & -13.82 & -14.6 & -17.79 \\
\hline
\end{tabular}


TABLE S3: Calculated Hyperfine Coupling Constants (MHz) of $\alpha$-protons in $\mathrm{Car}^{+}$, $\mathrm{Car}^{+}$in water, \#Car.(5), \#Car.(9) and \#Car. (13) using B3PW91/TZP(Ahlrichs)//B3LYP/6-31G**.

\begin{tabular}{|c|c|c|c|c|c|}
\hline Position & $\mathrm{Car}^{+}$ & $\begin{array}{l}\mathrm{Car}^{+} \text {in } \\
\text { water }\end{array}$ & \#Car·(5) & \#Car·(9) & \#Car·(13) \\
\hline \multirow[t]{2}{*}{4} & 9.26 & 8.43 & -1.16 & -0.38 & -0.36 \\
\hline & 12.14 & 10.82 & -0.06 & -0.31 & -0.34 \\
\hline \multirow[t]{2}{*}{$4^{\prime}$} & 12.14 & 10.83 & 4.28 & 4.75 & 5.99 \\
\hline & 9.27 & 8.44 & 4.36 & 5.03 & 6.38 \\
\hline 7 & -8.29 & -8.42 & 2.76 & 0.86 & 0.64 \\
\hline $7^{\prime}$ & -8.29 & -8.42 & -8.27 & -9.06 & -11.91 \\
\hline 8 & 1.73 & 1.99 & -7.48 & -1.54 & -0.18 \\
\hline $\mathbf{8}^{\prime}$ & 1.73 & 1.98 & 2.69 & 2.95 & 4.41 \\
\hline 10 & 0.54 & 1.04 & -9.97 & -11.27 & -0.35 \\
\hline $10^{\prime}$ & 0.54 & 1.03 & 4.35 & 4.70 & 6.75 \\
\hline 11 & -6.88 & -7.47 & 4.69 & 4.22 & 1.74 \\
\hline 11' & -6.88 & -7.46 & -12.95 & -13.89 & -17.27 \\
\hline 12 & 0.64 & 1.03 & -11.86 & -11.58 & -2.67 \\
\hline 12' & 0.64 & 1.02 & 5.83 & 6.24 & 8.56 \\
\hline 14 & -1.81 & -1.65 & -14.14 & -14.45 & -18.37 \\
\hline $14^{\prime}$ & -1.82 & -1.66 & 6.60 & 6.80 & 8.62 \\
\hline 15 & -3.21 & -3.41 & 6.64 & 6.66 & 7.60 \\
\hline $15^{\prime}$ & -3.21 & -3.39 & -15.51 & -16.07 & -18.16 \\
\hline
\end{tabular}


TABLE S4: Calculated Hyperfine Coupling Constant Tensors (MHz) of Methyl Protons in $\mathrm{Car}^{+}$, Car ${ }^{+}$in water, \#Car.(5), \#Car.(9) and \#Car- (13) using B3LYP/TZP(Ahlrichs)//B3LYP/3-21G.

\begin{tabular}{|c|c|c|c|c|c|c|}
\hline Position & Parameter & Car $^{+}$ & $\begin{array}{c}\text { Car }^{+} \text {in } \\
\text { water }\end{array}$ & \#Car.(5) & \#Car.(9) & \#Car.(13) \\
\hline \multirow[t]{4}{*}{$5 '$} & $\mathrm{~A}_{11}$ & 4.81 & 4.16 & 1.15 & 1.40 & 1.99 \\
\hline & $\mathrm{A}_{22}$ & 5.28 & 4.62 & 1.54 & 1.80 & 2.49 \\
\hline & $\mathrm{A}_{33}$ & 6.37 & 5.62 & 2.20 & 2.56 & 3.41 \\
\hline & $\mathbf{A}_{\text {iso }}$ & 5.49 & 4.80 & 1.63 & 1.92 & 2.63 \\
\hline \multirow[t]{4}{*}{$9^{\prime}$} & $\mathrm{A}_{11}$ & 5.92 & 6.55 & 6.93 & 7.72 & 9.62 \\
\hline & $\mathrm{A}_{22}$ & 6.75 & 7.41 & 7.91 & 8.78 & 10.86 \\
\hline & $\mathrm{A}_{33}$ & 7.98 & 8.69 & 9.22 & 10.23 & 12.58 \\
\hline & $\mathbf{A}_{\text {iso }}$ & 6.88 & 7.55 & 8.02 & 8.91 & 11.02 \\
\hline \multirow[t]{4}{*}{ 13' } & $\mathrm{A}_{11}$ & 3.70 & 4.37 & 10.62 & 11.46 & 13.13 \\
\hline & $\mathrm{A}_{22}$ & 4.51 & 5.21 & 11.99 & 12.89 & 14.72 \\
\hline & $\mathrm{A}_{33}$ & 5.38 & 6.14 & 13.72 & 14.74 & 16.79 \\
\hline & $\mathbf{A}_{\text {iso }}$ & 4.53 & 5.24 & 12.11 & 13.03 & 14.88 \\
\hline \multirow[t]{4}{*}{5} & $\mathrm{~A}_{11}$ & 4.81 & 4.16 & -1.53 & -0.28 & -0.18 \\
\hline & $\mathrm{A}_{22}$ & 5.28 & 4.62 & -0.79 & -0.19 & -0.11 \\
\hline & $\mathrm{A}_{33}$ & 6.37 & 5.62 & -0.12 & 0.17 & 0.02 \\
\hline & $\mathbf{A}_{\text {iso }}$ & 5.49 & 4.80 & -0.81 & -0.10 & -0.09 \\
\hline \multirow[t]{4}{*}{9} & $\mathrm{~A}_{11}$ & 5.92 & 6.55 & -3.31 & -9.47 & -0.94 \\
\hline & $\mathrm{A}_{22}$ & 6.75 & 7.41 & -3.09 & -5.93 & -0.76 \\
\hline & $\mathrm{A}_{33}$ & 7.98 & 8.69 & -2.33 & -1.67 & -0.46 \\
\hline & $\overline{\mathbf{A}_{\text {iso }}}$ & 6.88 & 7.55 & -2.91 & -5.69 & -0.72 \\
\hline \multirow[t]{4}{*}{13} & $\mathrm{~A}_{11}$ & 3.70 & 4.37 & -5.69 & -5.59 & -19.31 \\
\hline & $\mathrm{A}_{22}$ & 4.51 & 5.21 & -5.43 & -5.24 & -12.11 \\
\hline & $\mathrm{A}_{33}$ & 5.38 & 6.14 & -4.48 & -4.33 & -3.97 \\
\hline & $\overline{\mathbf{A}_{\text {iso }}}$ & 4.53 & 5.24 & -5.20 & -5.05 & -11.8 \\
\hline
\end{tabular}


TABLE S5: Calculated Hyperfine Coupling Constant Tensors (MHz) of Methyl Protons in $\mathrm{Car}^{+}, \mathrm{Car}^{+}$in water, \#Car.(5), \#Car.(9) and \#Car- (13) using B3PW91/TZP(Ahlrichs)//B3LY/3-21G.

\begin{tabular}{|c|c|c|c|c|c|c|}
\hline Position & Parameter & $\mathrm{Car}^{+}$ & $\begin{array}{l}\text { Car }^{+} \text {in } \\
\text { water }\end{array}$ & \#Car.(5) & \#Car·(9) & \#Car·(13) \\
\hline \multirow[t]{4}{*}{$5^{\prime}$} & $\mathrm{A}_{11}$ & 4.85 & 4.20 & 1.18 & 1.46 & 2.08 \\
\hline & $\mathrm{A}_{22}$ & 5.32 & 4.66 & 1.57 & 1.87 & 2.59 \\
\hline & $\mathrm{A}_{33}$ & 6.45 & 5.69 & 2.26 & 2.67 & 3.55 \\
\hline & $\mathbf{A}_{\text {iso }}$ & 5.54 & 4.85 & 1.67 & 2.00 & 2.74 \\
\hline \multirow[t]{4}{*}{ 9' } & $\mathrm{A}_{11}$ & 5.93 & 6.56 & 7.06 & 8.00 & 9.92 \\
\hline & $\mathrm{A}_{22}$ & 6.78 & 7.43 & 8.05 & 9.09 & 11.20 \\
\hline & $\mathrm{A}_{33}$ & 7.99 & 8.69 & 9.37 & 10.54 & 12.90 \\
\hline & $\mathbf{A}_{\text {iso }}$ & 6.90 & 7.56 & 8.16 & 9.21 & 11.34 \\
\hline \multirow[t]{4}{*}{ 13' } & $\mathrm{A}_{11}$ & 3.66 & 4.30 & 10.70 & 11.69 & 13.34 \\
\hline & $\mathrm{A}_{22}$ & 4.46 & 5.14 & 12.07 & 13.12 & 14.96 \\
\hline & $\mathrm{A}_{33}$ & 5.32 & 6.05 & 13.76 & 14.92 & 16.94 \\
\hline & $\mathbf{A}_{\text {iso }}$ & 4.48 & 5.16 & 12.18 & 13.24 & 15.08 \\
\hline \multirow[t]{4}{*}{5} & $\mathrm{~A}_{11}$ & 4.85 & 4.20 & -1.67 & -0.32 & -1.85 \\
\hline & $\mathrm{A}_{22}$ & 5.32 & 4.66 & -0.90 & -0.22 & -0.23 \\
\hline & $\mathrm{A}_{33}$ & 6.45 & 5.69 & -0.19 & 0.15 & 1.75 \\
\hline & $\overline{\mathbf{A}_{\text {iso }}}$ & 5.54 & 4.85 & -0.92 & -0.13 & -0.11 \\
\hline \multirow[t]{4}{*}{9} & $\mathrm{~A}_{11}$ & 5.93 & 6.56 & -3.51 & -10.57 & -1.10 \\
\hline & $\mathrm{A}_{22}$ & 6.78 & 7.43 & -3.31 & -6.67 & -0.89 \\
\hline & $\mathrm{A}_{33}$ & 7.99 & 8.69 & -2.54 & -2.20 & -0.59 \\
\hline & $\mathbf{A}_{\text {iso }}$ & 6.90 & 7.56 & -3.12 & -6.48 & -0.86 \\
\hline \multirow[t]{4}{*}{13} & $\mathrm{~A}_{11}$ & 3.66 & 4.30 & -5.98 & -6.04 & -21.53 \\
\hline & $\mathrm{A}_{22}$ & 4.46 & 5.14 & -5.76 & -5.73 & -13.65 \\
\hline & $\mathrm{A}_{33}$ & 5.32 & 6.05 & -4.82 & -4.85 & -5.12 \\
\hline & $\mathbf{A}_{\text {iso }}$ & 4.48 & 5.16 & -5.52 & -5.54 & -13.43 \\
\hline
\end{tabular}


TABLE S6: Calculated Hyperfine Coupling Constant Tensors (MHz) of Methyl Protons in $\mathrm{Car}^{+}$, Car ${ }^{+}$in water, \#Car.(5), \#Car.(9) and \#Car- (13) using B3LYP/TZP(Ahlrichs)//SVWN5/6-31G*.

\begin{tabular}{|c|c|c|c|c|c|c|}
\hline Position & Parameter & $\mathrm{Car}^{+}$ & $\begin{array}{l}\text { Car }^{+} \text {in } \\
\text { water }\end{array}$ & \#Car·(5) & \#Car·(9) & \#Car·(13) \\
\hline \multirow[t]{4}{*}{$5^{\prime}$} & $\mathrm{A}_{11}$ & 6.98 & 6.80 & 4.24 & 4.64 & 5.90 \\
\hline & $\mathrm{A}_{22}$ & 7.41 & 7.20 & 4.64 & 5.10 & 6.38 \\
\hline & $\mathrm{A}_{33}$ & 8.78 & 8.56 & 5.61 & 6.18 & 7.70 \\
\hline & $\mathbf{A}_{\text {iso }}$ & 7.72 & 7.52 & 4.83 & 5.30 & 6.66 \\
\hline \multirow[t]{4}{*}{$9^{\prime}$} & $\mathrm{A}_{11}$ & 6.07 & 6.95 & 8.23 & 9.36 & 10.86 \\
\hline & $\mathrm{A}_{22}$ & 6.91 & 7.82 & 9.29 & 10.50 & 12.16 \\
\hline & $\mathrm{A}_{33}$ & 8.02 & 8.98 & 10.50 & 11.85 & 13.70 \\
\hline & $\overline{A_{\text {iso }}}$ & 7.00 & 7.92 & 9.34 & 10.57 & 12.24 \\
\hline \multirow[t]{4}{*}{ 13' } & $\mathrm{A}_{11}$ & 3.67 & 4.32 & 10.85 & 11.98 & 13.30 \\
\hline & $\mathrm{A}_{22}$ & 4.44 & 5.11 & 12.14 & 13.36 & 14.84 \\
\hline & $\mathrm{A}_{33}$ & 5.21 & 5.90 & 13.46 & 14.78 & 16.47 \\
\hline & $\mathbf{A}_{\text {iso }}$ & 4.44 & 5.11 & 12.15 & 13.37 & 14.87 \\
\hline \multirow[t]{4}{*}{5} & $\mathrm{~A}_{11}$ & 6.87 & 6.64 & -4.93 & -0.79 & -0.53 \\
\hline & $\mathrm{A}_{22}$ & 7.31 & 7.06 & -3.09 & -0.64 & -0.40 \\
\hline & $\mathrm{A}_{33}$ & 8.65 & 8.38 & -0.86 & -0.13 & -0.28 \\
\hline & $\overline{\mathbf{A}_{\text {iso }}}$ & 7.61 & 7.36 & -2.96 & -0.52 & -0.40 \\
\hline \multirow[t]{4}{*}{9} & $\mathrm{~A}_{11}$ & 6.11 & 7.01 & -4.48 & -13.72 & -1.44 \\
\hline & $\mathrm{A}_{22}$ & 6.95 & 7.89 & -4.27 & -8.55 & -1.18 \\
\hline & $\mathrm{A}_{33}$ & 8.06 & 9.04 & -3.53 & -2.60 & -0.86 \\
\hline & $\mathbf{A}_{\text {iso }}$ & 7.04 & 7.98 & -4.09 & -8.29 & -1.16 \\
\hline \multirow[t]{4}{*}{13} & $\mathrm{~A}_{11}$ & 3.74 & 4.41 & -6.43 & -6.62 & -22.34 \\
\hline & $\mathrm{A}_{22}$ & 4.51 & 5.21 & -6.25 & -6.29 & -13.96 \\
\hline & $\mathrm{A}_{33}$ & 5.28 & 6.01 & -5.47 & -5.57 & -4.53 \\
\hline & $\mathbf{A}_{\text {iso }}$ & 4.51 & 5.21 & -6.05 & -6.16 & -13.61 \\
\hline
\end{tabular}


TABLE S7: Calculated Hyperfine Coupling Constant Tensors (MHz) of Methyl Protons in $\mathrm{Car}^{+}$, Car ${ }^{+}$in water, \#Car.(5), \#Car.(9) and \#Car- (13) using B3PW91/TZP(Ahlrichs)//SVWN5/6-31G*.

\begin{tabular}{|c|c|c|c|c|c|c|}
\hline Position & Parameter & Car $^{+}$ & $\begin{array}{c}\text { Car }^{+} \text {in } \\
\text { water }\end{array}$ & $\# \operatorname{Car} \cdot(5)$ & \#Car·(9) & \#Car.(13) \\
\hline \multirow[t]{4}{*}{$5^{\prime}$} & $\mathrm{A}_{11}$ & 6.90 & 6.98 & 4.35 & 4.82 & 6.04 \\
\hline & $\mathrm{A}_{22}$ & 7.32 & 7.37 & 4.75 & 5.27 & 6.51 \\
\hline & $\mathrm{A}_{33}$ & 8.72 & 8.80 & 5.78 & 6.41 & 7.91 \\
\hline & $\mathbf{A}_{\text {iso }}$ & 7.64 & 7.71 & 4.96 & 5.50 & 6.82 \\
\hline \multirow[t]{4}{*}{ 9' } & $\mathrm{A}_{11}$ & 5.96 & 7.06 & 8.43 & 9.66 & 11.05 \\
\hline & $\mathrm{A}_{22}$ & 6.80 & 7.94 & 9.51 & 10.82 & 12.38 \\
\hline & $\mathrm{A}_{33}$ & 7.91 & 9.09 & 10.71 & 12.16 & 13.89 \\
\hline & $\mathbf{A}_{\text {iso }}$ & 6.89 & 8.03 & 9.55 & 10.88 & 12.44 \\
\hline \multirow[t]{4}{*}{$13^{\prime}$} & $\mathrm{A}_{11}$ & 3.55 & 4.29 & 11.69 & 12.21 & 13.41 \\
\hline & $\mathrm{A}_{22}$ & 4.31 & 5.08 & 13.00 & 13.61 & 14.97 \\
\hline & $\mathrm{A}_{33}$ & 5.07 & 5.84 & 14.28 & 14.96 & 16.53 \\
\hline & $\mathbf{A}_{\text {iso }}$ & 4.31 & 5.07 & 12.99 & 13.59 & 14.97 \\
\hline \multirow[t]{4}{*}{5} & $\mathrm{~A}_{11}$ & 6.79 & 6.83 & -4.77 & -0.88 & -0.61 \\
\hline & $\mathrm{A}_{22}$ & 7.22 & 7.23 & -3.76 & -0.71 & -0.46 \\
\hline & $\mathrm{A}_{33}$ & 8.58 & 8.62 & -1.40 & -0.18 & -0.32 \\
\hline & $\mathbf{A}_{\text {iso }}$ & 7.53 & 7.56 & -3.31 & -0.59 & -0.46 \\
\hline \multirow[t]{4}{*}{9} & $\mathrm{~A}_{11}$ & 6.00 & 7.14 & -4.80 & -15.18 & -1.65 \\
\hline & $\mathrm{A}_{22}$ & 6.84 & 8.03 & -4.62 & -9.54 & -1.34 \\
\hline & $\mathrm{A}_{33}$ & 7.94 & 9.17 & -3.87 & -3.30 & -1.03 \\
\hline & $\mathbf{A}_{\text {iso }}$ & 6.93 & 8.11 & -4.43 & -9.34 & -1.34 \\
\hline \multirow[t]{4}{*}{13} & $\mathrm{~A}_{11}$ & 3.61 & 4.39 & -6.83 & -7.14 & -24.43 \\
\hline & $\mathrm{A}_{22}$ & 4.38 & 5.19 & -6.67 & -6.84 & -15.40 \\
\hline & $\mathrm{A}_{33}$ & 5.15 & 5.96 & -5.91 & -6.15 & -5.62 \\
\hline & $\mathbf{A}_{\text {iso }}$ & 4.38 & 5.18 & -6.47 & -6.71 & -15.15 \\
\hline
\end{tabular}


TABLE S8: Calculated Hyperfine Coupling Constant Tensors (MHz) of Methyl Protons in $\mathrm{Car}^{+}, \mathrm{Car}^{+}$in water, \#Car.(5), \#Car.(9) and \#Car- (13) using B3LYP/TZP(Ahlrichs)//BPW91/DGDZVP2.

\begin{tabular}{|c|c|c|c|c|c|c|}
\hline Position & Parameter & $\mathrm{Car}^{+}$ & $\begin{array}{l}\text { Car }^{+} \text {in } \\
\text { water }\end{array}$ & \#Car·(5) & \#Car·(9) & \#Car·(13) \\
\hline \multirow[t]{4}{*}{$5^{\prime}$} & $\mathrm{A}_{11}$ & 6.26 & 5.58 & 2.65 & 3.09 & 3.96 \\
\hline & $\mathrm{A}_{22}$ & 6.71 & 6.02 & 3.08 & 3.53 & 4.47 \\
\hline & $\mathrm{A}_{33}$ & 8.04 & 7.24 & 3.90 & 4.44 & 5.58 \\
\hline & $\mathbf{A}_{\text {iso }}$ & 7.00 & 6.28 & 3.21 & 3.69 & 4.67 \\
\hline \multirow[t]{4}{*}{$9^{\prime}$} & $\mathrm{A}_{11}$ & 6.25 & 6.75 & 7.96 & 8.45 & 10.09 \\
\hline & $\mathrm{A}_{22}$ & 7.06 & 7.59 & 8.96 & 9.52 & 11.32 \\
\hline & $\mathrm{A}_{33}$ & 8.26 & 8.82 & 10.29 & 10.92 & 12.97 \\
\hline & $\mathbf{A}_{\text {iso }}$ & 7.19 & 7.72 & 9.07 & 9.63 & 11.46 \\
\hline \multirow[t]{4}{*}{ 13' } & $\mathrm{A}_{11}$ & 3.63 & 4.22 & 10.69 & 11.18 & 12.68 \\
\hline & $\mathrm{A}_{22}$ & 4.38 & 5.00 & 11.96 & 12.51 & 14.18 \\
\hline & $\mathrm{A}_{33}$ & 5.16 & 5.84 & 13.44 & 14.05 & 15.96 \\
\hline & $\mathbf{A}_{\text {iso }}$ & 4.39 & 5.02 & 12.03 & 12.58 & 14.27 \\
\hline \multirow[t]{4}{*}{5} & $\mathrm{~A}_{11}$ & 6.26 & 5.58 & -2.88 & -0.55 & -0.37 \\
\hline & $\mathrm{A}_{22}$ & 6.71 & 6.02 & -1.76 & -0.41 & -0.26 \\
\hline & $\mathrm{A}_{33}$ & 8.04 & 7.24 & -0.41 & 0.03 & -0.12 \\
\hline & $\overline{\mathbf{A}_{\text {iso }}}$ & 7.00 & 6.28 & -1.68 & -0.31 & -0.25 \\
\hline \multirow[t]{4}{*}{9} & $\mathrm{~A}_{11}$ & 6.25 & 6.75 & -1.24 & -13.93 & -1.31 \\
\hline & $\mathrm{A}_{22}$ & 7.06 & 7.59 & -1.05 & -8.55 & -1.06 \\
\hline & $\mathrm{A}_{33}$ & 8.26 & 8.82 & 1.00 & -2.57 & -0.75 \\
\hline & $\mathbf{A}_{\text {iso }}$ & 7.19 & 7.72 & -0.43 & -8.35 & -1.04 \\
\hline \multirow[t]{4}{*}{13} & $\mathrm{~A}_{11}$ & 3.63 & 4.22 & -6.34 & -6.35 & -22.10 \\
\hline & $\mathrm{A}_{22}$ & 4.38 & 5.00 & -6.15 & -6.03 & -13.66 \\
\hline & $\mathrm{A}_{33}$ & 5.16 & 5.84 & -5.36 & -5.29 & -4.34 \\
\hline & $\mathbf{A}_{\text {iso }}$ & 4.39 & 5.02 & -5.95 & -5.89 & -13.37 \\
\hline
\end{tabular}


TABLE S9: Calculated Hyperfine Coupling Constant Tensors (MHz) of Methyl Protons in $\mathrm{Car}^{+}$, Car ${ }^{+}$in water, \#Car.(5), \#Car.(9) and \#Car- (13) using B3PW91/TZP(Ahlrichs)//BPW91/DGDZVP2.

\begin{tabular}{|c|c|c|c|c|c|c|}
\hline Position & Parameter & $\mathrm{Car}^{+}$ & $\begin{array}{l}\text { Car }^{+} \text {in } \\
\text { water }\end{array}$ & \#Car.(5) & \#Car·(9) & \#Car·(13) \\
\hline \multirow[t]{4}{*}{$5^{\prime}$} & $\mathrm{A}_{11}$ & 6.12 & 5.61 & 2.78 & 3.48 & 4.07 \\
\hline & $\mathrm{A}_{22}$ & 6.57 & 6.04 & 3.21 & 3.93 & 4.58 \\
\hline & $\mathrm{A}_{33}$ & 7.90 & 7.31 & 4.09 & 4.95 & 5.75 \\
\hline & $\mathbf{A}_{\text {iso }}$ & 6.86 & 6.32 & 3.36 & 4.12 & 4.80 \\
\hline \multirow[t]{4}{*}{$9^{\prime}$} & $\mathrm{A}_{11}$ & 6.07 & 6.73 & 8.27 & 9.28 & 10.30 \\
\hline & $\mathrm{A}_{22}$ & 6.88 & 7.58 & 9.30 & 10.39 & 11.56 \\
\hline & $\mathrm{A}_{33}$ & 8.08 & 8.79 & 10.63 & 11.81 & 13.18 \\
\hline & $\mathbf{A}_{\text {iso }}$ & 7.01 & 7.70 & 9.40 & 10.49 & 11.68 \\
\hline \multirow[t]{4}{*}{ 13' } & $\mathrm{A}_{11}$ & 3.54 & 4.14 & 10.91 & 11.9 & 12.80 \\
\hline & $\mathrm{A}_{22}$ & 4.29 & 4.92 & 12.19 & 13.27 & 14.32 \\
\hline & $\mathrm{A}_{33}$ & 5.07 & 5.73 & 13.62 & 14.7 & 16.04 \\
\hline & $\mathbf{A}_{\text {iso }}$ & 4.30 & 4.93 & 12.24 & 13.29 & 14.39 \\
\hline \multirow[t]{4}{*}{5} & $\mathrm{~A}_{11}$ & 6.12 & 5.61 & -3.26 & -0.72 & -0.42 \\
\hline & $\mathrm{A}_{22}$ & 6.56 & 6.04 & -2.04 & -0.53 & -0.29 \\
\hline & $\mathrm{A}_{33}$ & 7.90 & 7.31 & -0.58 & -0.06 & -0.16 \\
\hline & $\overline{\mathbf{A}_{\text {iso }}}$ & 6.86 & 6.32 & -1.96 & -0.44 & -0.29 \\
\hline \multirow[t]{4}{*}{9} & $\mathrm{~A}_{11}$ & 6.07 & 6.73 & -1.24 & -17.19 & -1.47 \\
\hline & $\mathrm{A}_{22}$ & 6.88 & 7.58 & -0.97 & -10.80 & -1.18 \\
\hline & $\mathrm{A}_{33}$ & 8.08 & 8.79 & 1.21 & -3.97 & -0.88 \\
\hline & $\mathbf{A}_{\text {iso }}$ & 7.01 & 7.70 & -0.33 & -10.65 & -1.18 \\
\hline \multirow[t]{4}{*}{13} & $\mathrm{~A}_{11}$ & 3.54 & 4.14 & -6.86 & -7.54 & -24.16 \\
\hline & $\mathrm{A}_{22}$ & 4.29 & 4.92 & -6.69 & -7.29 & -15.07 \\
\hline & $\mathrm{A}_{33}$ & 5.07 & 5.73 & -5.95 & -6.63 & -5.41 \\
\hline & $\mathbf{A}_{\text {iso }}$ & 4.30 & 4.93 & -6.50 & -7.15 & -14.88 \\
\hline
\end{tabular}


TABLE S10: Calculated Hyperfine Coupling Constant Tensors (MHz) of Methyl Protons in $\mathrm{Car}^{+}$, Car ${ }^{+}$in water, \#Car.(5), \#Car.(9) and \#Car. (13) using B3LYP/TZP(Ahlrichs)//B3LYP/DGDZVP2.

\begin{tabular}{|c|c|c|c|c|c|c|}
\hline Position & Parameter & $\mathrm{Car}^{+}$ & $\begin{array}{l}\text { Car }^{+} \text {in } \\
\text { water }\end{array}$ & \#Car.(5) & \#Car·(9) & \#Car·(13) \\
\hline \multirow[t]{4}{*}{$5^{\prime}$} & $\overline{\mathrm{A}_{11}}$ & 5.57 & 5.54 & 2.43 & 2.63 & 3.26 \\
\hline & $\mathrm{A}_{22}$ & 6.02 & 5.96 & 2.86 & 3.06 & 3.75 \\
\hline & $\mathrm{A}_{33}$ & 7.25 & 7.22 & 3.69 & 3.94 & 4.78 \\
\hline & $\mathbf{A}_{\text {iso }}$ & 6.28 & 6.24 & 2.99 & 3.21 & 3.93 \\
\hline \multirow[t]{4}{*}{ 9' } & $\mathrm{A}_{11}$ & 6.38 & 7.73 & 9.12 & 9.34 & 10.79 \\
\hline & $\mathrm{A}_{22}$ & 7.21 & 8.61 & 10.18 & 10.46 & 12.04 \\
\hline & $\mathrm{A}_{33}$ & 8.44 & 9.91 & 11.57 & 11.89 & 13.69 \\
\hline & $\mathbf{A}_{\text {iso }}$ & 7.34 & 8.75 & 10.29 & 10.57 & 12.17 \\
\hline \multirow[t]{4}{*}{ 13' } & $\mathrm{A}_{11}$ & 3.81 & 4.76 & 12.62 & 13.02 & 14.12 \\
\hline & $\mathrm{A}_{22}$ & 4.58 & 5.57 & 13.99 & 14.44 & 15.68 \\
\hline & $\mathrm{A}_{33}$ & 5.41 & 6.41 & 15.57 & 16.07 & 17.54 \\
\hline & $\mathbf{A}_{\text {iso }}$ & 4.60 & 5.58 & 14.06 & 14.51 & 15.78 \\
\hline \multirow[t]{4}{*}{5} & $\mathrm{~A}_{11}$ & 5.57 & 5.54 & -2.95 & -0.66 & -0.53 \\
\hline & $\mathrm{A}_{22}$ & 6.02 & 5.96 & -1.88 & -0.49 & -0.37 \\
\hline & $\mathrm{A}_{33}$ & 7.25 & 7.22 & -0.60 & -0.04 & -0.21 \\
\hline & $\overline{\mathbf{A}_{\text {iso }}}$ & 6.28 & 6.24 & -1.81 & -0.40 & -0.37 \\
\hline \multirow[t]{4}{*}{9} & $\mathrm{~A}_{11}$ & 6.38 & 7.73 & -5.12 & -16.23 & -2.03 \\
\hline & $\mathrm{A}_{22}$ & 7.21 & 8.61 & -4.98 & -10.37 & -1.67 \\
\hline & $\mathrm{A}_{33}$ & 8.44 & 9.91 & -4.27 & -3.85 & -1.37 \\
\hline & $\mathbf{A}_{\text {iso }}$ & 7.34 & 8.75 & -4.79 & -10.15 & -1.69 \\
\hline \multirow[t]{4}{*}{13} & $\mathrm{~A}_{11}$ & 3.81 & 4.76 & -7.97 & -7.89 & -26.72 \\
\hline & $\mathrm{A}_{22}$ & 4.58 & 5.57 & -7.85 & -7.64 & -17.18 \\
\hline & $\mathrm{A}_{33}$ & 5.41 & 6.41 & -7.09 & -6.93 & -6.62 \\
\hline & $\mathbf{A}_{\text {iso }}$ & 4.60 & 5.58 & -7.64 & -7.49 & -16.84 \\
\hline
\end{tabular}


TABLE S11: Calculated Hyperfine Coupling Constant Tensors (MHz) of Methyl Protons in $\mathrm{Car}^{+}$, Car ${ }^{+}$in water, \#Car.(5), \#Car.(9) and \#Car- (13) using B3PW91/TZP(Ahlrichs)//B3LYP/DGDZVP2.

\begin{tabular}{|c|c|c|c|c|c|c|}
\hline Position & Parameter & Car $^{+}$ & $\begin{array}{l}\mathrm{Car}^{+} \text {in } \\
\text { water }\end{array}$ & \#Car·(5) & \#Car.(9) & \#Car·(13) \\
\hline \multirow[t]{4}{*}{$5^{\prime}$} & $\overline{A_{11}}$ & 6.23 & 5.94 & 2.56 & 2.94 & 3.37 \\
\hline & $\mathrm{A}_{22}$ & 6.66 & 6.35 & 3.00 & 3.38 & 3.87 \\
\hline & $\mathrm{A}_{33}$ & 8.05 & 7.72 & 3.90 & 4.35 & 4.97 \\
\hline & $\mathbf{A}_{\text {iso }}$ & 6.98 & 6.67 & 3.15 & 3.56 & 4.07 \\
\hline \multirow[t]{4}{*}{ 9' } & $\mathrm{A}_{11}$ & 6.99 & 8.04 & 9.47 & 9.84 & 11.07 \\
\hline & $A_{22}$ & 7.85 & 8.95 & 10.56 & 10.97 & 12.35 \\
\hline & $\mathrm{A}_{33}$ & 9.10 & 10.25 & 11.95 & 12.40 & 14.00 \\
\hline & $\mathbf{A}_{\text {iso }}$ & 7.98 & 9.08 & 10.66 & 11.07 & 12.47 \\
\hline \multirow[t]{4}{*}{$13^{\prime}$} & $\overline{A_{11}}$ & 3.88 & 4.78 & 12.87 & 13.14 & 14.30 \\
\hline & $\mathrm{A}_{22}$ & 4.65 & 5.58 & 14.26 & 14.58 & 15.89 \\
\hline & $\mathrm{A}_{33}$ & 5.42 & 6.39 & 15.77 & 16.11 & 17.66 \\
\hline & $\overline{\mathbf{A}_{\text {iso }}}$ & 4.65 & 5.58 & 14.30 & 14.61 & 15.95 \\
\hline \multirow[t]{4}{*}{5} & $\mathrm{~A}_{11}$ & 6.23 & 5.94 & -3.32 & -0.89 & -0.60 \\
\hline & $\mathrm{A}_{22}$ & 6.66 & 6.35 & -2.16 & -0.67 & -0.39 \\
\hline & $\mathrm{A}_{33}$ & 8.05 & 7.72 & -0.76 & -0.18 & -0.25 \\
\hline & $\mathbf{A}_{\text {iso }}$ & 6.98 & 6.67 & -2.08 & -0.58 & -0.40 \\
\hline \multirow[t]{4}{*}{9} & $\mathrm{~A}_{11}$ & 6.99 & 8.04 & -5.53 & -19.74 & -2.19 \\
\hline & $A_{22}$ & 7.85 & 8.95 & -5.42 & -12.80 & -1.79 \\
\hline & $\mathrm{A}_{33}$ & 9.10 & 10.25 & -4.71 & -5.41 & -1.51 \\
\hline & $\mathbf{A}_{\text {iso }}$ & 7.98 & 9.08 & -5.22 & -12.65 & -1.83 \\
\hline \multirow[t]{4}{*}{13} & $\mathrm{~A}_{11}$ & 3.88 & 4.78 & -8.55 & -8.70 & -29.25 \\
\hline & $A_{22}$ & 4.65 & 5.58 & -8.44 & -8.51 & -18.91 \\
\hline & $\mathrm{A}_{33}$ & 5.42 & 6.39 & -7.74 & -7.87 & -7.92 \\
\hline & $\mathbf{A}_{\text {iso }}$ & 4.65 & 5.58 & -8.24 & -8.36 & -18.69 \\
\hline
\end{tabular}


TABLE S12: Calculated Spin Density Distributions of $\mathrm{Car}^{+}$, \#Car•(5), \#Car•(9) and \#Car•(13) using B3LYP/TZP(Ahlrichs)//B3LYP/6-31G**.

\begin{tabular}{|c|c|c|c|c|c|c|c|c|c|c|c|}
\hline & C5 & C6 & C7 & $\mathrm{C8}$ & C9 & C10 & C11 & C12 & C13 & C14 & C15 \\
\hline Car $^{+}$ & 0.10 & -0.04 & 0.15 & -0.05 & 0.14 & -0.04 & 0.12 & -0.03 & 0.09 & 0.01 & 0.05 \\
\hline \#Car॰(5) & -0.02 & 0.11 & -0.05 & 0.13 & -0.08 & 0.18 & -0.11 & 0.21 & -0.13 & 0.25 & -0.15 \\
\hline \#Car॰(9) & -0.003 & 0.002 & -0.01 & 0.01 & -0.06 & 0.19 & -0.10 & 0.21 & -0.13 & 0.26 & -0.15 \\
\hline$\# \mathbf{C}$ & -0.005 & 0.003 & -0.02 & 0.01 & -0.02 & 0.02 & -0.04 & 0.04 & -0.12 & 0.32 & -0.18 \\
\hline & $\mathrm{C15}^{\prime}$ & C14' & $\mathrm{C13}^{\prime}$ & C12' & C11' & $\mathrm{C10}^{\prime}$ & C9' & $\mathrm{C8}^{\prime}$ & C7' & C6' $^{\prime}$ & $\mathrm{C5}^{\prime}$ \\
\hline Car & 0.05 & 0.01 & 0.09 & -0.03 & 0.12 & -0.04 & 0.14 & -0.05 & 0.14 & -0.04 & 0.10 \\
\hline \#Car•(5) & 0.28 & -0.15 & 0.27 & -0.14 & 0.23 & -0.11 & 0.18 & -0.07 & 0.14 & -0.03 & 0.04 \\
\hline \#Car॰(9) & 0.29 & -0.16 & 0.29 & -0.15 & 0.25 & -0.12 & 0.20 & -0.08 & 0.15 & -0.03 & 0.05 \\
\hline \#Car॰(13) & 0.32 & -0.20 & 0.34 & -0.20 & 0.31 & -0.16 & 0.26 & -0.11 & 0.20 & -0.05 & 0.07 \\
\hline
\end{tabular}


TABLE S13: Calculated Spin Density Distributions of $\mathrm{Car}^{+}$, \#Car•(5), \#Car•(9) and \#Car•(13) using B3PW91/ TZP(Ahlrichs)//B3LYP/6-31G**

\begin{tabular}{|c|c|c|c|c|c|c|c|c|c|c|c|}
\hline & C5 & C6 & C7 & C8 & C9 & C10 & C11 & C12 & C13 & C14 & C15 \\
\hline Car·+ & 0.11 & -0.04 & 0.16 & -0.06 & 0.15 & -0.04 & 0.13 & -0.03 & 0.09 & 0.01 & 0.05 \\
\hline \#Car॰(5) & -0.02 & 0.12 & -0.06 & 0.15 & -0.09 & 0.20 & -0.13 & 0.23 & -0.15 & 0.27 & -0.18 \\
\hline \#Car•(9) & -0.004 & 0.003 & -0.02 & 0.01 & -0.07 & 0.21 & -0.12 & 0.23 & -0.15 & 0.28 & -0.18 \\
\hline \#Car & -0.004 & 0.002 & -0.01 & 0.01 & -0.02 & 0.02 & -0.04 & 0.03 & -0.13 & 0.32 & -0.19 \\
\hline & $\mathrm{C}^{\prime} 5^{\prime}$ & C14' & C13' & C12' & C11' & $\mathrm{ClO}^{\prime}$ & C9' & C8' & C7' & C6' & C5' \\
\hline Car $^{+}$ & 0.05 & 0.01 & 0.09 & -0.03 & 0.13 & -0.04 & 0.15 & -0.06 & 0.16 & -0.04 & 0.11 \\
\hline \#Car•(5) & 0.30 & -0.18 & 0.29 & -0.16 & 0.25 & -0.13 & 0.20 & -0.08 & 0.15 & -0.04 & 0.05 \\
\hline \#Car•(9) & 0.31 & -0.19 & 0.31 & -0.17 & 0.27 & -0.13 & 0.22 & -0.09 & 0.16 & -0.04 & 0.06 \\
\hline \#Car•(13) & 0.33 & -0.21 & 0.35 & -0.21 & 0.32 & -0.17 & 0.26 & -0.12 & 0.21 & -0.05 & 0.07 \\
\hline
\end{tabular}


TABLE S14: Total Electronic Energy (a.u.) of Car ${ }^{+}$, \#Car.(5), \#Car.(9) and \#Car· (13) with DFT Methods.

\begin{tabular}{|l|c|c|c|c|}
\hline DFT Method & Car $^{+}$ & \#Car·(5) & \#Car·(9) & \#Car• (13) $^{+}$ \\
\hline B3LYP / 3-21G & -1549.221579 & -1548.787746 & -1548.778452 & -1548.775586 \\
\hline SVWN5 / 6-31G* & -1543.248248 & -1542.818303 & -1542.360313 & -1542.803872 \\
\hline BPW91 / DGDZVP2 & -1557.779841 & -1557.343076 & -1557.331247 & -1557.327877 \\
\hline B3LYP / 6-31G** & -1557.793530 & -1557.354579 & -1557.345959 & -1557.342979 \\
\hline B3LYP / DGDZVP2 & -1558.039473 & -1557.607525 & -1557.597401 & -1557.594692 \\
\hline
\end{tabular}


TABLE S15: Bond Lengths (Angstrom) and Angles (Degrees) for $\beta$-carotene Radical Cation and Neutral Radicals Obtained with B3LYP/3-21G.

\begin{tabular}{lrrrr}
\hline Parameter & Car $^{+}$ & \#Car·(5) & \#Car·(9) & \#Car·(13) \\
\hline C1-C2 & 1.551 & 1.560 & 1.550 & 1.551 \\
C2-C3 & 1.535 & 1.541 & 1.536 & 1.536 \\
C3-C4 & 1.535 & 1.552 & 1.537 & 1.537 \\
C4-C5 & 1.518 & 1.531 & 1.523 & 1.523 \\
C5-C6 & 1.364 & 1.486 & 1.351 & 1.351 \\
C6-C7 & 1.456 & 1.359 & 1.481 & 1.480 \\
C7-C8 & 1.367 & 1.439 & 1.344 & 1.349 \\
C8-C9 & 1.436 & 1.372 & 1.484 & 1.460 \\
C9-C10 & 1.389 & 1.440 & 1.468 & 1.362 \\
C10-C11 & 1.408 & 1.372 & 1.362 & 1.443 \\
C11-C12 & 1.386 & 1.420 & 1.427 & 1.352 \\
C12-C13 & 1.413 & 1.387 & 1.381 & 1.477 \\
C13-C14 & 1.402 & 1.422 & 1.428 & 1.463 \\
C14-C15 & 1.396 & 1.387 & 1.382 & 1.368 \\
C15-C15' & 1.396 & 1.403 & 1.407 & 1.419 \\
C15'-C14' & 1.396 & 1.397 & 1.394 & 1.385 \\
C14'-C13' & 1.402 & 1.398 & 1.401 & 1.410 \\
C13'-C12' & 1.413 & 1.422 & 1.419 & 1.413 \\
C12'-C11' & 1.386 & 1.377 & 1.379 & 1.384 \\
C11'-C10' & 1.408 & 1.423 & 1.422 & 1.418 \\
C10'-C9' & 1.389 & 1.375 & 1.376 & 1.379 \\
C9'-C8' & 1.436 & 1.451 & 1.450 & 1.448 \\
C8'-C7' & 1.367 & 1.355 & 1.355 & 1.357 \\
C7'-C6' & 1.456 & 1.477 & 1.477 & 1.475 \\
C6'-C5' & 1.364 & 1.353 & 1.353 & 1.354 \\
C5'-C4' & 1.518 & 1.523 & 1.522 & 1.522 \\
C4'-C3' & 1.535 & 1.537 & 1.537 & 1.537 \\
C3'-C2' & 1.535 & 1.536 & 1.536 & 1.536 \\
C2'-C1' & 1.551 & 1.551 & 1.551 & 1.551
\end{tabular}




$\begin{array}{lrrrr}\text { < C5-C6-C7 } & 122.965 & 123.249 & 122.314 & 122.314 \\ \text { < C5-C6-C7-C8 } & -36.273 & -5.264 & -53.339 & -53.355 \\ \text { < C5'-C6'-C7' } & 122.964 & 122.496 & 122.693 & 122.612 \\ \text { < C5'-C6'-C7'-C8' } & 36.270 & -50.147 & -49.398 & -47.739\end{array}$


TABLE S16: Bond Lengths (Angstrom) and Angles (Degrees) for $\beta$-carotene Radical Cation and Neutral Radicals Obtained with SVWN5/6-31G*

\begin{tabular}{lrrrr}
\hline Parameter & Car $^{+}$ & \#Car·(5) & \#Car·(9) & \#Car·(13) \\
\hline C1-C2 & 1.528 & 1.531 & 1.526 & 1.527 \\
C2-C3 & 1.509 & 1.513 & 1.510 & 1.510 \\
C3-C4 & 1.510 & 1.519 & 1.510 & 1.511 \\
C4-C5 & 1.490 & 1.511 & 1.495 & 1.495 \\
C5-C6 & 1.375 & 1.458 & 1.362 & 1.363 \\
C6-C7 & 1.431 & 1.372 & 1.449 & 1.447 \\
C7-C8 & 1.373 & 1.412 & 1.352 & 1.360 \\
C8-C9 & 1.418 & 1.381 & 1.458 & 1.434 \\
C9-C10 & 1.390 & 1.416 & 1.445 & 1.370 \\
C10-C11 & 1.397 & 1.377 & 1.366 & 1.419 \\
C11-C12 & 1.384 & 1.401 & 1.407 & 1.358 \\
C12-C13 & 1.404 & 1.389 & 1.384 & 1.453 \\
C13-C14 & 1.397 & 1.406 & 1.411 & 1.443 \\
C14-C15 & 1.390 & 1.384 & 1.381 & 1.368 \\
C15-C15' & 1.390 & 1.394 & 1.397 & 1.404 \\
C15'-C14' & 1.390 & 1.390 & 1.387 & 1.382 \\
C14'-C13' & 1.397 & 1.395 & 1.397 & 1.402 \\
C13'-C12' & 1.404 & 1.407 & 1.405 & 1.401 \\
C12'-C11' & 1.384 & 1.380 & 1.381 & 1.384 \\
C11'-C10' & 1.397 & 1.404 & 1.403 & 1.400 \\
C10'-C9' & 1.390 & 1.382 & 1.382 & 1.384 \\
C9'-C8' & 1.418 & 1.425 & 1.424 & 1.423 \\
C8'-C7' & 1.373 & 1.366 & 1.366 & 1.368 \\
C7'-C6' & 1.431 & 1.443 & 1.443 & 1.442 \\
C6'-C5' & 1.375 & 1.366 & 1.366 & 1.366 \\
C5'-C4' & 1.490 & 1.495 & 1.494 & 1.494 \\
C4'-C3' & 1.510 & 1.511 & 1.511 & 1.511 \\
C3'-C2' & 1.509 & 1.510 & 1.509 & 1.510
\end{tabular}




$\begin{array}{lrrrr}\text { C2'-C1' } & 1.528 & 1.528 & 1.526 & 1.528 \\ \text { < C5-C6-C7 } & 123.268 & 122.877 & 122.676 & 123.227 \\ \text { < C5-C6-C7-C8 } & -25.497 & -9.633 & -34.863 & -33.592 \\ \text { < C5'-C6'-C7' } & 123.509 & 123.501 & 123.023 & 123.436 \\ \text { < C5'-C6'-C7'-C8' } & 22.738 & -30.106 & -32.114 & -29.828\end{array}$


TABLE S17: Bond Lengths (Angstrom) and Angles (Degrees) for $\beta$-carotene Radical Cation and Neutral Radicals Obtained with BPW91/DGDZVP2

\begin{tabular}{|c|c|c|c|c|}
\hline Parameter & Car $^{+}$ & \#Car·(5) & \#Car·(9) & \#Car·(13) \\
\hline $\mathrm{C} 1-\mathrm{C} 2$ & 1.549 & 1.556 & 1.549 & 1.548 \\
\hline $\mathrm{C} 2-\mathrm{C} 3$ & 1.530 & 1.536 & 1.531 & 1.531 \\
\hline $\mathrm{C} 3-\mathrm{C} 4$ & 1.530 & 1.544 & 1.532 & 1.532 \\
\hline $\mathrm{C} 4-\mathrm{C} 5$ & 1.511 & 1.528 & 1.518 & 1.518 \\
\hline $\mathrm{C} 5-\mathrm{C} 6$ & 1.384 & 1.482 & 1.370 & 1.371 \\
\hline C6-C7 & 1.453 & 1.381 & 1.476 & 1.473 \\
\hline $\mathrm{C} 7-\mathrm{C} 8$ & 1.384 & 1.434 & 1.360 & 1.369 \\
\hline C8-C9 & 1.435 & 1.392 & 1.481 & 1.454 \\
\hline C9-C10 & 1.404 & 1.436 & 1.464 & 1.383 \\
\hline $\mathrm{C} 10-\mathrm{C} 11$ & 1.413 & 1.391 & 1.381 & 1.439 \\
\hline $\mathrm{C} 11-\mathrm{C} 12$ & 1.399 & 1.420 & 1.425 & 1.370 \\
\hline $\mathrm{C} 12-\mathrm{C} 13$ & 1.421 & 1.403 & 1.400 & 1.473 \\
\hline C13-C14 & 1.412 & 1.424 & 1.428 & 1.462 \\
\hline C14-C15 & 1.406 & 1.400 & 1.397 & 1.384 \\
\hline C15-C15' & 1.406 & 1.411 & 1.413 & 1.421 \\
\hline C15'-C14' & 1.406 & 1.406 & 1.404 & 1.398 \\
\hline C14'-C13' & 1.412 & 1.410 & 1.412 & 1.417 \\
\hline C13'-C12' & 1.421 & 1.424 & 1.423 & 1.419 \\
\hline C12'-C11' & 1.399 & 1.394 & 1.395 & 1.399 \\
\hline C11'-C10' & 1.413 & 1.423 & 1.422 & 1.419 \\
\hline C10'-C9' & 1.404 & 1.394 & 1.395 & 1.398 \\
\hline C9'-C8' & 1.435 & 1.445 & 1.444 & 1.443 \\
\hline $\mathrm{C} 8^{\prime}-\mathrm{C} 7^{\prime}$ & 1.384 & 1.375 & 1.376 & 1.377 \\
\hline C7'-C6' & 1.453 & 1.469 & 1.468 & 1.467 \\
\hline C6'-C5' & 1.384 & 1.373 & 1.374 & 1.374 \\
\hline C5'-C4' & 1.511 & 1.516 & 1.516 & 1.516 \\
\hline $\mathrm{C}^{\prime}-\mathrm{C} 3^{\prime}$ & 1.530 & 1.532 & 1.531 & 1.531 \\
\hline $\mathrm{C} 3^{\prime}-\mathrm{C} 2^{\prime}$ & 1.530 & 1.531 & 1.531 & 1.531 \\
\hline
\end{tabular}




$\begin{array}{lrrrr}\text { C2'-C1' } & 1.549 & 1.549 & 1.549 & 1.549 \\ \text { < C5-C6-C7 } & 123.211 & 123.000 & 122.492 & 122.851 \\ \text { < C5-C6-C7-C8 } & -30.971 & -7.004 & -45.488 & -43.149 \\ \text { < C5'-C6'-C7' } & 123.211 & 122.754 & 123.071 & 123.151 \\ \text { < C5'-C6'-C7'-C8' } & 30.973 & -40.915 & -39.47 & -38.202\end{array}$


TABLE S18: The Hyperfine Coupling Constants (MHz) for the $\beta$-carotene Radical Cation $\mathrm{Car}^{+}{ }^{+}$using B3LYP/TZP(Ahlrichs)//B3LYP/6-31G**. Small values $<0.1 \mathrm{MHz}$ are not included. Only $\mathrm{A}_{\text {iso }}$ is given for the methyl groups.

\begin{tabular}{|c|c|c|c|c|c|}
\hline Position & $\begin{array}{c}\text { No. of } \\
\text { protons }\end{array}$ & $\mathbf{A}_{\mathbf{x x}}$ & $\overline{A_{y y}}$ & $\overline{\mathbf{A}_{\mathrm{zz}}}$ & $\mathbf{A}_{\text {iso }}$ \\
\hline \multirow[t]{2}{*}{1} & 3 & & & & 0.007 \\
\hline & 3 & & & & -0.038 \\
\hline \multirow[t]{2}{*}{$1^{\prime}$} & 3 & & & & -0.036 \\
\hline & 3 & & & & 0.008 \\
\hline \multirow[t]{2}{*}{2} & 1 & -0.132 & -0.064 & 0.439 & 0.081 \\
\hline & 1 & -0.333 & -0.246 & 0.6 & 0.007 \\
\hline \multirow[t]{2}{*}{$2^{\prime}$} & 1 & -0.13 & -0.062 & 0.441 & 0.083 \\
\hline & 1 & -0.334 & -0.248 & 0.6 & 0.006 \\
\hline \multirow[t]{2}{*}{3} & 1 & -0.355 & -0.324 & 0.295 & -0.128 \\
\hline & 1 & -0.409 & -0.307 & 0.608 & -0.036 \\
\hline \multirow[t]{2}{*}{$3^{\prime}$} & 1 & -0.357 & -0.326 & 0.293 & -0.130 \\
\hline & 1 & -0.412 & -0.31 & 0.604 & -0.039 \\
\hline \multirow[t]{2}{*}{4} & 1 & 8.54 & 8.83 & 10.21 & 9.19 \\
\hline & 1 & 11.38 & 11.61 & 13.19 & 12.06 \\
\hline \multirow[t]{2}{*}{$44^{\prime}$} & 1 & 11.38 & 11.61 & 13.19 & 12.06 \\
\hline & 1 & 8.54 & 8.83 & 10.21 & 9.19 \\
\hline 5 & 3 & & & & 5.89 \\
\hline $5^{\prime}$ & 3 & & & & 5.89 \\
\hline 7 & 1 & -12.04 & -8.09 & -2.85 & -7.66 \\
\hline $7^{\prime}$ & 1 & -12.04 & -8.09 & -2.85 & -7.66 \\
\hline 8 & 1 & -0.05 & 1.07 & 3.33 & 1.45 \\
\hline $8^{\prime}$ & 1 & -0.05 & 1.07 & 3.33 & 1.45 \\
\hline 9 & 3 & & & & 7.05 \\
\hline $9^{\prime}$ & 3 & & & & 7.05 \\
\hline 10 & 1 & -0.93 & 0.02 & 1.93 & 0.34 \\
\hline
\end{tabular}




\begin{tabular}{|c|c|c|c|c|c|}
\hline $10^{\prime}$ & 1 & -0.93 & 0.02 & 1.93 & 0.34 \\
\hline 11 & 1 & -9.66 & -7.1 & -2.53 & -6.43 \\
\hline $11^{\prime}$ & 1 & -9.66 & -7.1 & -2.53 & -6.43 \\
\hline 12 & 1 & -0.8 & 0.02 & 2.1 & 0.44 \\
\hline $12^{\prime}$ & 1 & -0.8 & 0.02 & 2.1 & 0.44 \\
\hline 13 & 3 & & & & 4.6 \\
\hline $13^{\prime}$ & 3 & & & & 4.6 \\
\hline 14 & 1 & -2.87 & -2.72 & 0.16 & -1.81 \\
\hline $14^{\prime}$ & 1 & -2.88 & -2.72 & 0.15 & -1.82 \\
\hline 15 & 1 & -4.53 & -4.02 & -0.75 & -3.10 \\
\hline $15^{\prime}$ & 1 & -4.53 & -4.02 & -0.75 & -3.10 \\
\hline
\end{tabular}


TABLE S19: The Hyperfine Coupling Constants (MHz) for the $\beta$-carotene Radical \#Car·(5) using B3LYP/TZP(Ahlrichs)//B3LYP/6-31G**. Small values $<0.1 \mathrm{MHz}$ are not included. Only $\mathrm{A}_{\text {iso }}$ is given for the methyl groups.

\begin{tabular}{|c|c|c|c|c|c|}
\hline Position & $\begin{array}{l}\text { No. of } \\
\text { protons }\end{array}$ & $\mathbf{A}_{\mathbf{x x}}$ & $\mathbf{A}_{\mathrm{yy}}$ & $\mathbf{A}_{\mathrm{zz}}$ & $\mathbf{A}_{\text {iso }}$ \\
\hline \multirow[t]{2}{*}{1} & 3 & & & & 0.480 \\
\hline & 3 & & & & 0.169 \\
\hline \multirow[t]{2}{*}{$1^{\prime}$} & 3 & & & & 0.008 \\
\hline & 3 & & & & 0.034 \\
\hline \multirow[t]{2}{*}{2} & 1 & -0.357 & -0.341 & 0.461 & -0.079 \\
\hline & 1 & -0.34 & -0.325 & 0.252 & -0.138 \\
\hline \multirow[t]{2}{*}{$2^{\prime}$} & 1 & -0.197 & -0.141 & 0.365 & 0.009 \\
\hline & 1 & 0.027 & 0.089 & 0.445 & 0.187 \\
\hline \multirow[t]{2}{*}{3} & 1 & -0.174 & -0.17 & 0.281 & -0.021 \\
\hline & 1 & -0.136 & -0.096 & 0.814 & 0.194 \\
\hline \multirow[t]{2}{*}{$3^{\prime}$} & 1 & -0.22 & -0.164 & 0.33 & -0.018 \\
\hline & 1 & -0.182 & -0.163 & 0.192 & -0.051 \\
\hline \multirow[t]{2}{*}{4} & 1 & -1.295 & -1.164 & -0.511 & $\begin{array}{l}-0.99 \\
\end{array}$ \\
\hline & 1 & -0.294 & -0.166 & 0.429 & -0.01 \\
\hline \multirow[t]{2}{*}{$44^{\prime}$} & 1 & 3.73 & 3.88 & 4.57 & 4.06 \\
\hline & 1 & 3.81 & 3.84 & 4.77 & 4.14 \\
\hline 5 & 2 & -2.08 & $\begin{array}{l}-1.21 \\
\end{array}$ & -0.25 & \\
\hline $5^{\prime}$ & 3 & & & & 2.12 \\
\hline 7 & 1 & 1.25 & 1.89 & 3.64 & 2.26 \\
\hline
\end{tabular}




\begin{tabular}{|c|c|c|c|c|c|}
\hline $7^{\prime}$ & 1 & -11.14 & -7.48 & -3.16 & -7.26 \\
\hline 8 & 1 & -9.72 & -7.38 & -2.55 & -6.55 \\
\hline $8^{\prime}$ & 1 & 0.838 & 1.42 & 4.01 & 2.09 \\
\hline 9 & 3 & & & & -3.12 \\
\hline $9^{\prime}$ & 3 & & & & 8.44 \\
\hline 10 & 1 & -13.2 & -9.57 & -3.57 & -8.78 \\
\hline $10^{\prime}$ & 1 & 1.87 & 2.15 & 6.17 & 3.40 \\
\hline 11 & 1 & 2.18 & 2.39 & 6.45 & 3.67 \\
\hline $11^{\prime}$ & 1 & -17.57 & -11.81 & -4.97 & -11.45 \\
\hline 12 & 1 & -15.62 & -11.44 & -4.44 & -10.5 \\
\hline $12^{\prime}$ & 1 & 2.75 & 2.84 & 8.15 & 4.58 \\
\hline 13 & 3 & & & & -5.42 \\
\hline $13^{\prime}$ & 3 & & & & 12.48 \\
\hline 14 & 1 & -19.0 & -13.55 & -5.22 & -12.59 \\
\hline $14^{\prime}$ & 1 & 3.13 & 3.30 & 9.14 & 5.19 \\
\hline 15 & 1 & 3.26 & 3.32 & 9.05 & 5.21 \\
\hline $15^{\prime}$ & 1 & -21.22 & -14.53 & -5.71 & -13.82 \\
\hline
\end{tabular}


TABLE S20: The Hyperfine Coupling Constants (MHz) for the $\beta$-carotene Radical \#Car·(9) using B3LYP/TZP(Ahlrichs)//B3LYP/6-31G**. Small values $<0.1 \mathrm{MHz}$ are not included. Only $\mathrm{A}_{\text {iso }}$ is given for the methyl groups.

\begin{tabular}{|c|c|c|c|c|c|}
\hline Position & $\begin{array}{c}\text { No. of } \\
\text { protons }\end{array}$ & $\mathbf{A}_{\mathbf{x x}}$ & $\mathbf{A}_{\mathbf{y y}}$ & $\mathbf{A}_{\mathrm{zz}}$ & $\mathbf{A}_{\text {iso }}$ \\
\hline \multirow[t]{2}{*}{1} & 3 & & & & -0.004 \\
\hline & 3 & & & & -0.005 \\
\hline \multirow[t]{2}{*}{$1^{\prime}$} & 3 & & & & 0.011 \\
\hline & 3 & & & & 0.035 \\
\hline \multirow[t]{2}{*}{2} & 1 & -0.052 & -0.038 & 0.084 & -0.002 \\
\hline & 1 & -0.061 & -0.048 & 0.07 & -0.013 \\
\hline \multirow[t]{2}{*}{$2^{\prime}$} & 1 & -0.212 & -0.147 & 0.402 & 0.014 \\
\hline & 1 & 0.023 & 0.093 & 0.478 & 0.198 \\
\hline \multirow[t]{2}{*}{3} & 1 & -0.036 & -0.03 & 0.069 & 0.001 \\
\hline & 1 & -0.06 & -0.047 & 0.11 & 0.001 \\
\hline \multirow[t]{2}{*}{$3^{\prime}$} & 1 & -0.25 & -0.183 & 0.362 & -0.024 \\
\hline & 1 & -0.2 & -0.18 & 0.211 & -0.056 \\
\hline \multirow[t]{2}{*}{4} & 1 & -0.387 & -0.363 & -0.21 & -0.32 \\
\hline & & -0.288 & -0.277 & -0.184 & -0.25 \\
\hline \multirow[t]{2}{*}{$4^{\prime}$} & 1 & 4.22 & 4.37 & 5.15 & 4.58 \\
\hline & 1 & 4.47 & 4.51 & 5.54 & 4.84 \\
\hline 5 & 3 & & & & -0.17 \\
\hline $5^{\prime}$ & 3 & & & & 2.48 \\
\hline 7 & 1 & -0.09 & 0.27 & 1.99 & 0.72 \\
\hline $7^{\prime}$ & 1 & -12.42 & -8.38 & -3.6 & -8.13 \\
\hline 8 & 1 & -1.95 & -1.63 & -0.25 & -1.29 \\
\hline $8^{\prime}$ & 1 & 1.04 & 1.64 & 4.55 & 2.41 \\
\hline 9 & 2 & -10.26 & -6.380 & -1.810 & \\
\hline $9^{\prime}$ & 3 & & & & 9.23 \\
\hline 10 & 1 & -15.77 & -10.58 & -3.89 & -10.08 \\
\hline $10^{\prime}$ & 1 & 2.20 & 2.44 & 6.87 & 3.84 \\
\hline 11 & 1 & 1.7 & 2.07 & 6.55 & 3.44 \\
\hline
\end{tabular}




\begin{tabular}{|c|c|c|c|c|c|}
\hline $11^{\prime}$ & 1 & -19.15 & -12.94 & -5.53 & -12.54 \\
\hline 12 & 1 & -15.42 & -11.51 & -4.36 & -10.43 \\
\hline $12^{\prime}$ & 1 & 3.1 & 3.29 & 9.02 & 5.14 \\
\hline 13 & 3 & & & & $\mathbf{- 5 . 3 2}$ \\
\hline $13^{\prime}$ & 3 & & & & $\mathbf{1 3 . 3 1}$ \\
\hline 14 & 1 & -19.69 & -14.14 & -5.5 & -13.11 \\
\hline $14^{\prime}$ & 1 & 3.41 & 3.63 & 9.77 & 5.60 \\
\hline 15 & 1 & 3.48 & 3.52 & 9.44 & 5.48 \\
\hline $15^{\prime}$ & 1 & -22.3 & -15.38 & -6.12 & -14.6 \\
\hline
\end{tabular}


TABLE S21: The Hyperfine Coupling Constants (MHz) for the $\beta$-carotene Radical \#Car·(13) using B3LYP/TZP(Ahlrichs)//B3LYP/6-31G**. Small values $<0.1 \mathrm{MHz}$ are not included. Only $\mathrm{A}_{\text {iso }}$ is given for the methyl groups.

\begin{tabular}{|c|c|c|c|c|c|}
\hline Position & $\begin{array}{c}\text { No. of } \\
\text { protons }\end{array}$ & $\mathbf{A}_{\mathbf{x x}}$ & $\mathbf{A}_{\mathbf{y y}}$ & $\mathbf{A}_{\mathrm{zz}}$ & $\mathbf{A}_{\text {iso }}$ \\
\hline \multirow[t]{2}{*}{1} & 3 & & & & -0.006 \\
\hline & 3 & & & & -0.002 \\
\hline \multirow[t]{2}{*}{$1^{\prime}$} & 3 & & & & 0.004 \\
\hline & 3 & & & & 0.053 \\
\hline \multirow[t]{2}{*}{2} & 1 & -0.008 & 0.001 & 0.01 & 0.001 \\
\hline & 1 & -0.044 & -0.033 & -0.005 & -0.027 \\
\hline \multirow[t]{2}{*}{$2^{\prime}$} & 1 & -0.245 & -0.161 & 0.508 & 0.034 \\
\hline & 1 & 0.057 & 0.143 & 0.606 & 0.269 \\
\hline \multirow[t]{2}{*}{3} & 1 & -0.013 & -0.005 & 0.028 & 0.003 \\
\hline & 1 & -0.037 & -0.01 & 0.048 & 0.0003 \\
\hline \multirow[t]{2}{*}{$3^{\prime}$} & 1 & -0.331 & -0.246 & 0.43 & -0.049 \\
\hline & 1 & -0.279 & -0.254 & 0.234 & -0.10 \\
\hline \multirow[t]{2}{*}{4} & 1 & -0.482 & -0.417 & -0.361 & -0.42 \\
\hline & 1 & -0.45 & -0.404 & -0.375 & -0.41 \\
\hline \multirow[t]{2}{*}{$4^{\prime}$} & 1 & 5.72 & 5.9 & 6.91 & 6.18 \\
\hline & 1 & 6.17 & 6.22 & 7.53 & 6.64 \\
\hline 5 & 3 & & & & -0.21 \\
\hline $5^{\prime}$ & 3 & & & & 3.43 \\
\hline 7 & 1 & 0.282 & 0.771 & 1.38 & 0.81 \\
\hline $7^{\prime}$ & 1 & -17.69 & -12.25 & -5.88 & -11.94 \\
\hline 8 & 1 & -0.346 & -0.244 & -0.22 & -0.27 \\
\hline $8^{\prime}$ & 1 & 3.03 & 3.23 & 7.63 & 4.63 \\
\hline 9 & 3 & & & & -1.14 \\
\hline $9^{\prime}$ & 3 & & & & 12.03 \\
\hline 10 & 1 & -0.67 & -0.6 & -0.33 & -0.53 \\
\hline $10^{\prime}$ & 1 & 4.65 & 5.24 & 11.25 & 7.05 \\
\hline 11 & 1 & 0.19 & 1.26 & 4.64 & 2.03 \\
\hline
\end{tabular}




\begin{tabular}{|c|c|c|c|c|c|}
\hline $11^{\prime}$ & 1 & -24.99 & -17.36 & -8.29 & -16.88 \\
\hline 12 & 1 & -3.9 & -3.4 & -1.6 & -2.97 \\
\hline $12^{\prime}$ & 1 & 5.68 & 6.91 & 14.04 & 8.88 \\
\hline 13 & 2 & $-\mathbf{2 2 . 5 7}$ & $\mathbf{- 1 4 . 3 7}$ & $\mathbf{- 5 . 1 7}$ & \\
\hline $13^{\prime}$ & 3 & & & & $\mathbf{1 5 . 8 7}$ \\
\hline 14 & 1 & -27.75 & -18.95 & -7.9 & -18.20 \\
\hline $14^{\prime}$ & 1 & 5.73 & 6.84 & 14.25 & 8.94 \\
\hline 15 & 1 & 4.8 & 5.47 & 13.52 & 7.93 \\
\hline $15^{\prime}$ & 1 & -26.32 & -18.84 & -8.21 & -17.79 \\
\hline
\end{tabular}

